\title{
Towards simultaneous calibration-free and ultra-fast sensing of temperature and species in the intrapulse mode
}

Robin S.M. Chrystie*, Ehson F. Nasir, Aamir Farooq

Clean Combustion Research Center, Division of Physical Sciences and Engineering, King Abdullah University of Science and Technology (KAUST), Thuwal, 23955-6900, Saudi Arabia

*Corresponding author. Email: robin.chrystie@cantab.net, Phone: +966-128082704

Colloquium: Diagnostics

Total length: $\sim 5800$ words (Method 1)

Main text: 3700, equations: 99, references: 400, Figure 1: 190, Figure 2: 180, Figure 3: 207, Figure 4: 415, Figure 5: 297, Figure 6: 200, Figure 7: 180. 


\begin{abstract}
:
We report on exploiting the down-chirp phenomenon seen in quantum cascade lasers (QCLs), when modulated with long pulses, for the purpose of performing calibration-free and temporally resolved measurements. Intrapulse spectra of a native species (e.g. $\mathrm{H}_{2} \mathrm{O}$ ), common to combustion environments, were generated near $\lambda=7.62 \mu \mathrm{m}$ at repetition rates as high as $3.125 \mathrm{MHz}$. Two-line absorption spectroscopy was employed to infer calibration-free temperature from the chirp-induced intrapulse spectra. In this study, such temperature measurements were limited to rates of $250 \mathrm{kHz}$ due to spectral distortion at higher repetition rates. We demonstrate the ease at which accurate temperatures and $\mathrm{H}_{2} \mathrm{O}$ compositions can be achieved using simple and compact QCLs operated in the intrapulse mode. The sensor is also applicable to other species, and has the potential to be integrated into commercial technologies.
\end{abstract}

Keywords: Absorption; Calibration-free; Temperature sensing; Intrapulse; Shock tube. 


\section{Introduction}

Temporally-resolved in situ measurements of temperature and species [1-4] are essential to unveiling the transient physics of combustion processes. Applications benefiting from high time resolution include internal combustion engines (ICEs), pulsed detonation engines (PDEs), emerging deflagration-todetonation technology (DDT), supersonic combustion ramjet and shock tube research. Conventionally, fast sensors based on absorption spectroscopy use narrowband lasers fixed at a specific wavelength running in the $\mathrm{cw}$ mode $[3,5]$. This strategy is simple to implement and has a digital repetition rate typically limited by the detection system's sampling rate $(\sim \mathrm{MS} / \mathrm{s})$. However, the fixed wavelength approach is susceptible to potential inaccuracies induced by (i) drift and jitter of the laser, and (ii) evolution of composition and pressure of the system under measurement. Such changes result in uncertainties in the measured absorption, consequently incurring error in the inference of temperature and species concentration.

A wavelength scan across spectral lines overcomes this problem [6,7], rendering greater immunity to unknowns in linewidth and instrument spectral resolution. In the case of laser absorption spectroscopy, provided linestrengths are known a priori, the ensuing measurement of temperature and species is calibration-free. Attempts to concurrently harness both time resolution and calibration-free character, using rapid-scanned direct absorption, involve modulating the injection current of semiconductor lasers at high frequencies with either sawtooth or sinusoidal waveforms [8]. This method offers very good signal-to-noise capability, however, it suffers from an increasingly limited spectral modulation depth at higher frequencies, thus prohibiting the scan over complete line profiles. An alternative approach is that of the intrapulse technique $[7,9-11,14]$.

The intrapulse method exploits non quasi-steady-state thermally induced chirp that occurs as a result of modulating a semiconductor laser with a rectangular pulse train. The resulting chirp 
equates to a very rapid wavelength scan of the laser at typical rates of $\sim 100 \mathrm{MHz} / \mathrm{ns}$. If the pulse duration is set to hundreds of nanoseconds, then an ultra-rapid scan with sufficient spectral range can be achieved $\left(\sim 1-2 \mathrm{~cm}^{-1}\right)$, rendering the technique both time-resolved and calibration-free. Thus far, applications of this technique to monitor molecular species (e.g. $\left.\mathrm{NH}_{3}, \cdot \mathrm{CF}_{3}, \mathrm{CO}, \mathrm{COS}\right)$ comprise only a scant number of concentration measurements $[7,10,12]$ and rapid-passage $(\mathrm{RP})$ effect studies $[11,13,14]$.

A more complex technique that works on a similar principle involves the use of a supercontinuum light source. Although studies have shown promise, the restriction to the near-infrared wavelength region, as well as the inherent shot-to-shot fluctuations, reduces the SNR. This demands considerable bin-averages in excess of 1000 pulses, as seen in the work of Sanders [18] and Werblinski et al. [19], consequently compromising effective time resolutions of such sensors to well below $50 \mathrm{kHz}$. The typical chirp rates in these supercontinuum lasers are four orders of magnitude larger than that of chirped semiconductor lasers. This can result in unwanted coherence phenomena such as the rapid passage (RP) effect, which would therefore require larger pressures for dephasing to be guaranteed [14]. Furthermore, such large chirp rates result in very poor spectral resolutions of typically $\sim 5 \mathrm{~cm}^{-1}$.

To overcome past limitations, we demonstrate a novel sensor using a quantum cascade laser (QCL) in the intrapulse mode for probing a native species in combustion environments (e.g. $\mathrm{H}_{2} \mathrm{O}$ ). We demonstrate for the first time the promising potential of the intrapulse technique in being able to quantify temperature without necessitating calibration, whilst also achieving excellent time-resolution of up to $\sim 3 \mathrm{MHz}$ with quasi-instantaneous measurement times of $150 \mathrm{~ns}$. This is made possible through good SNR because of the near-perfect shot-to-shot stability, $30 \mathrm{~mW}$ pulse power, and laser wavelength corresponding to the stronger fundamental vibrational bands in the mid-infrared (near $7.62 \mu \mathrm{m}$ for $\mathrm{H}_{2} \mathrm{O}$ ). Preservation of time resolution is reconciled with good spectral resolution of $0.013 \mathrm{~cm}^{-1}$; the latter of which is possible through reasonable chirp rates, and therefore less likely to encounter the RP effect at 
pressures $[13,14]$ seen in combustion environments. This novel sensor is also easy with regards to implementation and data interpretation, is compact and uses only a single laser.

\section{Spectroscopy}

The recent development of QCLs has facilitated the application of absorption spectroscopy in the midinfrared; a spectral domain that is desired to be exploited due to stronger fundamental bands. The region corresponds to wavelengths of 3-15 $\mu \mathrm{m}$ with bands that typically exhibit absorbances 10-100 times more intense than that of the near-infrared. In this work, a spectral region for $\mathrm{H}_{2} \mathrm{O}$ near $1312 \mathrm{~cm}^{-1}(7.62 \mu \mathrm{m})$ was selected for the following reasons: (i) to avoid spectral interference from species in combustion environments such as $\mathrm{CO}, \mathrm{CO}_{2}$ and hydrocarbons, (ii) to achieve sufficient temperature sensitivity from the presence of two lines with a difference of lower-state energies exceeding $\Delta \mathrm{E} "=1000 \mathrm{~cm}^{-1}$, (iii) to use lines that possess sufficient linestrength at the conditions under study, and (iv) to be able to use a pulsed QCL that can access two closely-situated absorption features within a single laser pulse.

In this work, we base our novel sensor on the principle of laser absorption, where a laser beam is directed through a gaseous mixture of path length, L. Laser-based sensors can be used to determine the mole fraction of species of interest in a gas mixture using direct absorption spectroscopy (DAS), wherein the Beer-Lambert Law is used:

$$
I / I_{o}=\exp \left(-\alpha_{v}\right)
$$

Equation (1) relates the intensity transmitted through a gas medium, I, and the incident intensity, $\mathrm{I}_{\mathrm{o}}$, to the absorbance, $\alpha_{v}$, which itself is a function of the laser path length, L, the total pressure of the gas, P, 
the mole fraction of the absorbing species, $\mathrm{X}$, and spectroscopic parameters that make up the linestrength, $\mathrm{S}(\mathrm{T})$, and the lineshape function, $\phi_{v}(\mathrm{P}, \mathrm{T}, \mathrm{X})$ :

$$
\alpha_{v}=S(T) . P \cdot X \cdot L \cdot \phi_{v}(P, T, X)
$$

The incident and transmitted intensities are measured, which enables the determination of the mole fraction if other parameters are known. However, if a single absorption line is integrated with respect to wavelength, the need for knowledge of the broadening coefficients associated with $\phi_{v}(\mathrm{P}, \mathrm{T}, \mathrm{X})$ is removed, because the integral of the lineshape function is unity across a complete absorption feature. This can be exploited in practice by scanning a QCL across a wavelength range, recording the instantaneous absorbance, and then integrating the resulting spectra.

Using the scanning approach, temperature can also be computed by using the two-line equation:

$$
T=\frac{(h c / k)\left(E_{2}^{\prime \prime}-E_{1}^{\prime \prime}\right)}{\ln R+\ln \left(S_{2}\left(T_{o}\right) / S_{1}\left(T_{o}\right)\right)+(h c / k)\left(E_{2}^{\prime \prime}-E_{1}^{\prime \prime}\right) / T_{o}}
$$

where $\mathrm{h}, \mathrm{c}, \mathrm{k}, \mathrm{E}_{2}$ " and $\mathrm{E}_{1}$ " are constants. $\mathrm{S}\left(\mathrm{T}_{0}\right)$ is the reference linestrength at a temperature of $\mathrm{T}_{\mathrm{o}}=296 \mathrm{~K}$. Importantly, $\mathrm{R}$ is the ratio of linestrengths, $\mathrm{S}_{1}(\mathrm{~T}) / \mathrm{S}_{2}(\mathrm{~T})$, at temperature, $\mathrm{T}$, which is also equivalent to the ratio of integrated areas of the two corresponding spectral lines.

\section{Sensor Concept}

\subsection{Sensor architecture and application}

Figure 1 shows the experimental setup comprising a pulsed distributed feedback QCL (Alpes sb4332), with a scaled image showing the compact size of the laser head. The QCL was chosen due to its tunability across target $\mathrm{H}_{2} \mathrm{O}$ transitions from 1312 to $1316.4 \mathrm{~cm}^{-1}$. The emitted wavelength can be 
controlled by setting the laser head temperature and source voltage. Laser head temperature was regulated using a temperature controller (Alpes TCU200), while the voltage was regulated using a steady DC controller (GW Instek GPS-3030D). The timing of the pulsing (i.e. pulse duration and duty cycle) was regulated by a function generator (Lecroy WS2052). The laser head was mounted on an aluminium plate to dissipate any excess heat. The pulsed IR laser beam emerging from the head was split using a ZnSe plate, with the secondary reflected beam directed through a solid Germanium étalon (free spectral range (FSR): $494 \mathrm{MHz})$ towards a high bandwidth $(\sim 500 \mathrm{MHz})$ thermoelectrically-cooled photovoltaic detector (Vigo PVI 4TE-10.6-1x1-TO8- $\mathrm{BaF}_{2}$ ). The primary beam from the ZnSe plate was directed through a shock tube via $\mathrm{ZnSe}$ windows towards another similar fast detector. The path transversed through the shock tube corresponds to an inner diameter of $14.22 \mathrm{~cm}$. The experiments were performed in the stainless steel, low-pressure shock tube (LPST) facility at King Abdullah University of Science and Technology (KAUST). The shock tube is made of a $9 \mathrm{~m}$ driver section and a $9 \mathrm{~m}$ modular driven section. The incident shock speed is measured using a series of five piezoelectric PCB pressure transducers over the final $1.3 \mathrm{~m}$ of the shock tube. Reflected shock temperatures $\left(\mathrm{T}_{5}\right)$ and pressures $\left(\mathrm{P}_{5}\right)$ are determined from the measured incident shock speed and the standard 1-D shock-jump relations. Uncertainties in the calculated temperature and pressure are approximately $\pm 0.7 \%$ and $\pm 1 \%$, respectively.

The procedure for determining the instantaneous temperature of shock-heated gases inside the shock tube, using our intrapulse sensor, is as follows. Firstly, the shock tube was evacuated using vacuum pumps. The QCL is switched on and an appropriate voltage and laser head temperature is selected to obtain a wavelength near $7.62 \mu \mathrm{m}$ (close to the $\mathrm{H}_{2} \mathrm{O}$ transitions used in our experiment). The transmitted irradiance through the evacuated shock tube is recorded on an oscilloscope (Tektronix DPO3014, 2.5GS/s, $100 \mathrm{MHz}$ ), and this is used as the empty signal, $I_{o}$, as per the Beer-Lambert relation $\alpha_{v}=$ 
$\ln \left(I_{o} / I\right)$, where $\alpha_{v}$ is the absorbance and $I$ is the transmitted signal when the shock tube contains absorbing molecules. Thereafter, the driven section of the shock tube is filled with a gaseous mixture of fuel/oxidizer/argon, or neat $\mathrm{H}_{2} \mathrm{O} /$ argon, up to a low pressure of $\sim 50$ Torr, depending on the desired post reflected-shock conditions. Finally, the driver section of the shock tube is pressurized with helium gas until the diaphragm, that separates the driver and driven sections, ruptures. A shock wave consequently propagates through the driven section containing the low-pressure gaseous mixture. The absorption measurements are then carried out in the shock-heated gases at an optical location $2 \mathrm{~cm}$ away from the shock tube end-wall.

\subsection{The intrapulse approach}

The principle of the sensor developed in this work is based on chirp that occurs during a laser pulse. This effect is attributable to rapid transient heating of the laser chip observed in semi-conductor lasers when a step rise in injection current is applied. Figure 2 shows an example pulse at an instant in time superimposed on a flat baseline. The black trace shows an absorption feature embedded within the pulse as a result of passing the laser beam through a sample containing $\mathrm{H}_{2} \mathrm{O}$. In the absence of this feature, the profile of the pulse would otherwise be approximately flat and sloping, shown by the grey trace. The jagged nature at the edges of the pulse is due to detection system ringing, and therefore are not spectral features. The duration of the pulse in Fig. 2 is only $500 \mathrm{~ns}$. Each pulse can be repeated at rates of $\mathrm{kHz}$ to $\mathrm{MHz}$; in our study the maximum repetition rate was $3.125 \mathrm{MHz}$.

Intrapulse spectroscopy is rendered possible as a result of rapid frequency downchirp. This is manifested by the étalon profile depicted by the red trace in Fig. 2. The monotonic downchirp results in a spectral range of $\sim 0.8 \mathrm{~cm}^{-1}$ in this example, however the range can be controlled through (i) pulse timing from the function generator, (ii) regulation of the laser voltage, or (iii) laser head temperature. When the pulse duration is fixed, regulation of the voltage or head temperature effectively determines the degree of 
downchirp. For example, increasing the voltage results in a larger injection current, which results in a stronger degree of transient heating, and hence larger rates of chirp through the pulse. This would manifest as a more compressed étalon scan within the same pulse duration.

For many combustion applications, the temporal resolution of our sensor is excellent, as per the quasiinstantaneous nanosecond intrapulse timescales. However, spectral resolution within the pulse also needs to be accounted for. Spectral resolution is determined by the chirp rate $(\partial v / \partial t)$ at a given point within the pulse, as given by [17]:

$$
\Delta v_{r e s}=\beta(\partial v / \partial t)^{1 / 2}
$$

Here, $\beta$ is a constant, which is dependent on detection bandwidth. Chirp rates between 20 to $60 \mathrm{MHz} / \mathrm{ns}$ were seen in our experiments, giving rise to spectral resolutions of 380 to 620 $\mathrm{MHz}\left(0.013\right.$ to $\left.0.021 \mathrm{~cm}^{-1}\right)$, where $\beta=0.08$ for our system. It follows that increasing the chirp rate, increases the spectral width of the instrument function, and hence the spectral resolution decreases. Nonetheless, the spectral resolution for this sensor is similar to a high-end FTIR, yet has far better time resolution capability. The spectral resolution, $\Delta v_{\text {res }}$, is Gaussian [17], and so therefore was summed in quadrature to the Doppler width to correct the absorption profiles.

Accordingly, the effect of non-ideal monochromaticity from a measurable, yet small, spectral width requires that the applicability of the Beer-Lambert relation (Equation 1) is verified. This was performed by testing to see whether a measured absorbance $\left(\alpha_{v}\right)$, based on a typical spectral width of $0.013 \mathrm{~cm}^{-1}$ in this study, would scale in direct proportion with $\mathrm{H}_{2} \mathrm{O}$ mole fraction (X). It was assumed that the laser emits two separate beams of equal intensity $\mathrm{I}_{\mathrm{O}}$ that are separated by $0.013 \mathrm{~cm}^{-1}$. By considering the worst-case spectral condition of the beam pair being centered on the steepest flank of the $\mathrm{H}_{2} \mathrm{O}$ spectrum, with each wavenumber 
corresponding to the most dissimilar absorption cross-section combination, a slight non-linear relationship between $\alpha_{v}$ and $X$ was derived. This curvature is small enough, resuting in significant linearity for $\mathrm{H}_{2} \mathrm{O}$ mole fractions of interest in the current work $(\mathrm{X}<10 \%)$. From this conservative calculation, it was found that a $\sim 1 \%$ deviation in $\mathrm{H}_{2} \mathrm{O}$ mole fraction occurred when the mole fraction is 0.05 .

By recording incident and transmitted intensities, traces $I_{0}$ and $I$, an absorbance spectrum for a single pulse in the frequency domain can be computed; noting the laser exhibits negligible pulse-to-pulse fluctuation as observed on an oscilloscope. An example experimental intrapulse spectrum is shown by the red trace in Fig. 3 corresponding to $\mathrm{T}_{5}=1729 \mathrm{~K}, \mathrm{P}_{5}=1.29$ bar and $\mathrm{X}=1.2 \% \mathrm{H}_{2} \mathrm{O}$ in argon at laser settings of $250 \mathrm{kHz}$ repetition rate and chirp rate of $\sim 25 \mathrm{MHz} / \mathrm{ns}$. Absorbances are typically kept well below two units to maintain sensitive measurements. Using the HITRAN database [20], five lines are identified to be contributing to the entire spectrum, with energies, E”, given in Fig. 3. Using HITRAN parameters, and by accounting for spectral resolution, an overall spectral fit can be computed; this is depicted as the black trace in Fig. 3. The overall line fit is derived from the summation of the underlying Voigt profiles. Each Voigt profile can be simulated by floating both the peak and Lorentzian widths of the absorption transitions. The summation of these lines results in a fit that is computed from the Levenberg-Marquardt algorithm. In this study, we focused on the triplet of lines highlighted in Fig. 3. We used only these lines as they were sufficiently isolated from the neighboring pair, and the triplet possessed better temperature sensitivity due to the relatively smaller degree of overlap among the three lines. The corresponding nominal line positions are given by HITRAN as 1312.346, 1312.399 and $1312.418 \mathrm{~cm}^{-1}$.

\section{Results and Discussion}




\subsection{Cell measurements}

In order to infer temperatures accurately, reference linestrengths (i.e. $\left.\mathrm{S}_{\mathrm{o}}(296 \mathrm{~K})\right)$ within the HITRAN database require checking, since they can harbor errors in excess of 5\%. A custom-built high temperature optical cell under controlled conditions was employed. This cell consisted of Strontium Fluoride windows, allowing $7.62 \mu \mathrm{m}$ laser radiation to be passed efficiently. Pure water vapor was introduced into the cell at manometrically regulated pressures between $10-30$ Torr. The cell was placed within a tube furnace to allow the temperature of the water vapor inside the cell to be controlled, and measured using thermocouples. A cw external-cavity QCL was used to slowly scan and integrate across the spectral lines of interest to measure the underlying areas. The areas and pressures were used to infer linestrengths for a particular temperature, this process was repeated over a range of temperatures to accurately infer the reference linestrength at $296 \mathrm{~K}$. From this, we obtained improved estimates of $\mathrm{S}_{0}$, whose values agreed with HITRAN to $\pm 3 \%$.

\subsection{Shock tube validation measurements}

A number of non-reactive shocks using various $\mathrm{H}_{2} \mathrm{O} / \mathrm{Ar}$ mixtures were performed over a range of temperatures $(1095-1858 \mathrm{~K})$, pressures $\left(0.86-1.18\right.$ bar), and $\mathrm{H}_{2} \mathrm{O}$ mole fractions $(2.1-5.7 \%)$. Various laser settings were also investigated: repetition rates of $250 \mathrm{kHz}$ to $3.125 \mathrm{MHz}$, and chirp rates of 20 to $60 \mathrm{MHz} / \mathrm{ns}$. Figure 4 (a) shows a representative instantaneous spectrum at $\mathrm{T}_{5}=1560 \mathrm{~K}, \mathrm{P}_{5}$ $=1.04$ bar and $\mathrm{X}_{\mathrm{H} 2 \mathrm{O}}=2.1 \%$ peaking at an absorbance of 0.3 corresponding to an SNR of 20. Using Voigt profiles to fit the experimental spectrum, there is visibly good agreement when the laser settings are at $250 \mathrm{kHz}$ and $20 \mathrm{MHz} / \mathrm{ns}$. However, there is progressively less agreement between the measured and simulated profiles as repetition rate and chirp rate increase. This can be seen in Figs. 4 (b) and (c), where the simulated aggregate Voigt profile is unable to fit the measured spectrum satisfactorily. Here, the experimental spectrum is bin-averaged $(\mathrm{N}=10)$ to smoothen the spectrum, thus facilitating easy 
identification of subtle underlying spectral distortion. In Fig. 4 (b), the repetition rate is $1.25 \mathrm{MHz}$ and the chirp rate is $40 \mathrm{MHz} / \mathrm{ns}$. It can be seen under these conditions that a highly curved section near $v=$ 3.5 $\mathrm{GHz}$ is raised relative to an otherwise Voigt profile corresponding to the experimental conditions. Figure 4 (c) shows considerable spectral distortion at a repetition rate of $3.125 \mathrm{MHz}$ with a chirp rate of $60 \mathrm{MHz} / \mathrm{ns}$. It can now be seen that the small bump has grown in addition to the left flank of the large peak becoming less steep. It appears that with increasing chirp rate, the distortion from true Voigt profile worsens. Since repetition rate and chirp rate is coincidently correlated in our datasets, it is not immediately obvious which factor is the root cause of this problem; noting in principle that repetition rate and chirp rate can be set independently. Nonetheless, at $250 \mathrm{kHz}(20 \mathrm{MHz} / \mathrm{ns})$ the effect is irrelevant, as evinced in Fig. 4 (a), which is the laser setting used henceforth for quantifying temperature and $\mathrm{H}_{2} \mathrm{O}$ mole fraction. It should be noted that if the chirp rate is the sole cause of the undesired spectral distortion, then it would be possible to set the same laser to $\sim 2 \mathrm{MHz}$ with the smaller chirp rate of 20 $\mathrm{MHz} / \mathrm{ns}$, and therefore achieve improved (eightfold) temporal resolution capability.

Figure 5 (a) shows the temperature, as measured using the intrapulse method, with that derived from the 1-D shock relations. Each of the seven measured points were calculated by averaging 100 instantaneous spectra, and then fitting Voigt profiles, keeping the peak heights and Lorentzian widths as floating parameters. The temperature was inferred by integrating lines 1 and 2 and using Eq. (3). There is clearly good agreement between the measured temperatures and calculated temperature. The error bars represent the standard deviation over the number of spectra used in the averaging, with typical deviations of 2-3\%. Figure 5 (b) also shows good matching of the $\mathrm{H}_{2} \mathrm{O}$ mole fraction inferred from the intrapulse method with that from an independent measurement using a slow scanning laser $(5 \mathrm{kHz})$ in the near-infrared. 


\subsection{Demonstration in $\mathrm{H}_{2} / \mathrm{O}_{2} / \mathrm{Ar}$ shocks}

Hydrogen oxidation shocks were performed to test our novel sensor for the measurement of temperature and water mole fraction time-histories in transient combustion systems. Such a fuel (hydrogen) is already well researched, providing an accurate benchmark through established kinetic mechanisms (e.g. GRI [21]). Relatively high fuel concentration of $4 \%$ was used to cause appreciable temperature increase at the onset of ignition. Figure 6 is a raw time trace showing data recorded for about $400 \mu$ s, limited by the buffer size and resolution of the oscilloscope. The red trace in Fig. 6 shows the jump in pressure after the passing of the reflected shock. The blue trace depicts the train of transmission pulses recorded at a pulse repetition rate of $250 \mathrm{kHz}$ and chirp rate of $20 \mathrm{MHz} / \mathrm{ns}$. At the passing of the shock $(\mathrm{t}=0)$, there is a clear Schlieren perturbation, thereafter the transmission within each pulse clearly evolves with time, with a marked sudden change during $40<\mathrm{t}<80 \mu \mathrm{s}$, showing the region of ignition.

Figure 7 shows the corresponding time-history of temperature and $\mathrm{H}_{2} \mathrm{O}$ mole fraction at reflected-shock conditions of $\mathrm{T}_{5}=1519 \mathrm{~K}$ and $\mathrm{P}_{5}=0.92$ bar. The measurements are compared with the GRI mechanism [21] simulations conducted at constant volume and internal energy (constant UV); noting that this implementation leads to essentially the same results as compared to a constant pressure approach as the energy release is relatively small for the $4 \% \mathrm{H}_{2} / \mathrm{O}_{2} / \mathrm{Ar}$ mixture used here. For improved graphical clarity, data in Fig. 7 is binned $(\mathrm{N}=5)$ to $50 \mathrm{kHz}$ and the mole fraction trace is based on the measured average temperature. In spite of any noise in the traces of Fig. 7, there is reasonably good agreement between the well-established GRI mechanism for $\mathrm{H}_{2}$ oxidation and the intrapulse data, suggesting that the accuracy of our data is reasonable, with an associated temperature rise of $160 \mathrm{~K}$ and rise in mole fraction of $2.7 \%$ during the ignition event. The sensor can thus be used to accurately measure temperature and species concentration time-histories in reactive systems that require fast time response. 


\section{Summary and future work}

A novel absorption sensor based on an intrapulse approach has been developed, tested and applied to a shock tube. Such a sensor has shown promise to simultaneously capture calibration-free and highly time resolved temperature, as well as mole fraction data of a target absorbing molecule. Work is ongoing to address the issue of spectral distortion observed at more extreme laser settings explored here, namely at higher rates of chirp. Upon availability of quantum cascade lasers at an increasingly wider range of wavelengths, we endeavor to explore a more amenable case of two absorption lines with considerably less spectral interference. Nonetheless, our sensor shows great promise for the use of intrapulse technique to measure temperature and species, is applicable to species other than $\mathrm{H}_{2} \mathrm{O}$, and has the potential to be integrated into commercial technologies.

\section{Acknowledgements}

We are very thankful for funding provided by King Abdullah University of Science and Technology (KAUST) and the Clean Combustion Research Center (CCRC).

\section{References}

[1] R. K. Hanson, Proc. Combust. Inst. 33 (2011) 1.

[2] M. G. Allen, Meas. Sci. Technol. 9 (1998) 545.

[3] R. S. M. Chrystie, I. S. Burns, J. Hult, C. F. Kaminski, Opt. Lett. 34 (2009) 2492.

[4] R. S. M. Chrystie, I. S. Burns, C. F. Kaminski, Combust. Sci. Technol. 185 (2013) 180.

[5] H. Li, A. Farooq, J. B. Jeffries, R. K. Hanson, Appl. Phys. B. 89 (2007) 407.

[6] I. S. Burns, X. Mercier, M. Wartel, R. S. M. Chrystie, J. Hult, C. F. Kaminski, Proc. Combust. Inst. 33 (2011) 799.

[7] G. Wysocki, M. McCurdy, S. So, D. Weidmann, C. Roller, R. F. Curl, F. K. Tittel, Appl. Opt. 43 (2004) 6040. 
[8] V. Ebert, T. Fernholz, C. Giesemann, H. Pitz, H. Teichert, J. Wolfrum, H. Jaritz, J. Jeffries, C. Shaddix, Proc. Combust. Inst. 28 (2000) 423.

[9] L. A. Kranendonk, J. W. Walewski, T. Kim, S. T. Sanders, Proc. Combust. Inst. 30 (2005) 1619.

[10] E. Normand, M. McCulloch, G. Duxbury, N. Langford, Opt. Lett. 28 (2003) 16.

[11] J. A. Nwaboh, O. Werhahn, D. Schiel, Appl. Phys. B. 103 (2011) 947.

[12] J. Manne, W. Jager, J. Tulip, Appl. Phys. B. 94 (2009) 337.

[13] G. Hancock, S. J. Horrocks, G. A. D. Ritchie, J. H. van Helden, R. J. Walker, J. Phys. Chem. A. $112(2008) 9751$.

[14] J. H. Northern, G. A. D. Ritchie, E. P. Smakman, J. H. van Helden, J. Cockburn, G. Duxbury, Opt. Lett. 35 (2010) 2750.

[15] B. Grouiez, V. Zeninari, L. Joly, B. Parvitte, Appl. Phys. B. 100 (2010) 265.

[16] N. Tasinato, K. G. Hay, N. Langford, G. Duxbury, D. Wilson, J. Chem. Phys. 132 (2010).

[17] J. H. van Helden, S. J. Horrocks, G. A. D. Ritchie, Appl. Phys. Lett. 92 (2008).

[18] S. T. Sanders, Appl. Phys. B. 75 (2002) 799.

[19] T. Werblinski, S. R. Engel, R. Engelbrecht, L. Zigan, S. Will, Opt. Exp. 21 (2013) 13656.

[20] C. Richard, I. E. Gordon, L. S. Rothman, M. Abel, L. Frommhold, M. Gustafsson, J. M. Hartmann, C. Hermans, W. J. Lafferty, G. S. Orton, K. M. Smith, H. Tran, J. Quant. Spectrosc. Ra. 113 (2012) 1276.

[21] G. P. Smith, D. M. Golden, M. Frenklach, N. W. Moriarty, B. Eiteneer, M. Goldenberg, C. T. Bowman, R. K. Hanson, S. Song, W. C. Gardiner, Jr., V. V. Lissianski, and Z. Qin, available at $<$ http://www.me.berkeley.edu/gri mech/> 


\section{List of Figure Captions}

Fig. 1. Schematic of the setup for performing measurements using the intrapulse method. Grey lines denote electrical connections and red lines show the laser path. HBPD: High bandwidth photo diode, connected to an oscilloscope. The laser shown is a pulsed single-mode QCL.

Fig. 2. Internal structural detail of a pulse at the nanosecond timescale showing transmission in the presence of absorbing $\mathrm{H}_{2} \mathrm{O}$. The chirp present within the pulse is manifested by the étalon scan.

Fig. 3. Spectral lines within the range: 1312 to $1313 \mathrm{~cm}^{-1}$. Five lines of various lower level energies, E", of $\mathrm{H}_{2} \mathrm{O}$ are shown to form an aggregate spectrum. The boxed region portrays the relatively isolated triplet of lines used to infer temperature and mole fraction in this work. The span of the spectrum shown here corresponds to $300 \mathrm{~ns}$ of downchirp.

Fig. 4. Example spectral fits of the three lines of interest for $2.5 \% \mathrm{H}_{2} \mathrm{O} / \mathrm{Ar}$ at (a) $\mathrm{T}_{5}=1560 \mathrm{~K}, \mathrm{P}_{5}=1.04$ bar, $250 \mathrm{kHz}$ repetition rate; (b) $\mathrm{T}_{5}=1557 \mathrm{~K}, \mathrm{P}_{5}=0.94$ bar, $1.25 \mathrm{MHz}$ repetition rate; (c) $\mathrm{T}_{5}=1595 \mathrm{~K}$, $\mathrm{P}_{5}=0.95$ bar, $3.125 \mathrm{MHz}$ repetition rate.

Fig. 4. Temperature (a), and $\mathrm{H}_{2} \mathrm{O}$ molar composition (b), both inferred from intrapulse absorption spectra. These data are compared to 1-D shock relations and an independent scanning laser measurement respectively.

Fig. 5. Time trace after the reflected shock, showing pressure (red) and the QCL pulses at $250 \mathrm{kHz}$ (blue) for a $4 \% \mathrm{H}_{2} / \mathrm{O}_{2} / \mathrm{Ar}, \phi=1$ mixture with $\mathrm{T}_{5}=1519 \mathrm{~K}, \mathrm{P}_{5}=0.92$ bar. The transmittance within each pulse shows a decrease with time from about $40 \mu$ s after the shock front passing.

Fig. 6 . Measured temperature and $\mathrm{H}_{2} \mathrm{O}$ mole fraction during a shock with initial mixture: 4\% $\mathrm{H}_{2} / \mathrm{O}_{2} / \mathrm{Ar}$, $\phi=1, \mathrm{~T}_{5}=1519 \mathrm{~K}, \mathrm{P}_{5}=0.92$ bar recorded at $250 \mathrm{kHz}$, binned to $50 \mathrm{kHz}$. 